\title{
BMJ Open Supervised exercise delivered via telehealth in real time to manage chronic conditions in adults: a protocol for a scoping review to inform future research in stroke survivors
}

\author{
Emily R Ramage, ${ }^{\oplus 1,2}$ Natalie A Fini, ${ }^{\circledR 3,4}$ Elizabeth A Lynch, ${ }^{\odot 5}$ Amanda Patterson, ${ }^{1}$ \\ Catherine M Said, ${ }^{2,6}$ Coralie English $^{\oplus 1,7}$
}

To cite: Ramage ER, Fini NA, Lynch EA, et al. Supervised exercise delivered via telehealth in real time to manage chronic conditions in adults: a protocol for a scoping review to inform future research in stroke survivors. BMJ Open 2019;9:e027416. doi:10.1136/ bmjopen-2018-027416

- Prepublication history and additional material for this paper are available online. To view these files, please visit the journal online (http://dx.doi org/10.1136/bmjopen-2018027416).

Received 22 October 2018 Revised 13 December 2018 Accepted 7 January 2019

Check for updates

(C) Author(s) (or their employer(s)) 2019. Re-use permitted under CC BY-NC. No commercial re-use. See rights and permissions. Published by BMJ.

For numbered affiliations see end of article.

Correspondence to

Emily R Ramage;

emily.ramage@uon.edu.au

\section{ABSTRACT}

Introduction Increasing physical activity reduces secondary stroke risk factors, but many stroke survivors have low levels of physical activity. Supervised exercise delivered via telehealth has the potential to overcome barriers to increased physical activity in stroke survivors. Our scoping review will examine the emerging field of supervised exercise delivered via telehealth to map the available evidence in relation to its efficacy, acceptability, safety and feasibility in chronic conditions to inform future research into its ability to increase physical activity.

Methods and analysis The methodological framework of Arksey and 0'Malley will be applied to our scoping review. A systematic search of Medline, CINAHL, Scopus, Cochrane, Pedro and Embase; hand searching of pertinent studies' reference lists; and consultation with experts in the field will identify relevant papers. Studies involving participants with a chronic condition who undertake supervised exercise delivered by a health professional via telehealth targeted at improving secondary stroke risk factors or involving lower limb weight-bearing exercise will be included. Study selection and critical appraisal of individual studies will be carried out independently by two authors with discrepancies resolved by a third author. Quantitative and qualitative data will be charted using a standardised form. Results will be tabulated and narratively summarised to highlight findings relevant to the review's research questions and to inform recommendations for future research.

Ethics and dissemination Our review will significantly contribute to the knowledge base of exercise and rehabilitation delivered via telehealth and its application in chronic conditions, including stroke. Findings will be relevant to researchers, healthcare workers and policy-makers and will be disseminated through publication and presentations. Only secondary deidentified data will be included, therefore ethics approval will not be sought. This protocol is not registered as PROSPERO currently excludes scoping reviews.

\section{INTRODUCTION}

Stroke is the second leading cause of death and burden of disease worldwide. ${ }^{12}$ The number of people experiencing stroke is increasing, with estimates predicting 70 million stroke
Strengths and limitations of this study:

- Our comprehensive scoping review will bring together research findings regarding supervised exercise delivered via telehealth to inform its future application to research and practice across chronic conditions.

- The inclusion of all research study designs will ensure the breadth of evidence regarding supervised exercise delivered via telehealth is captured.

- We will use a published framework to optimise transparency and methodological rigour.

- To facilitate accurate analysis of the evidence and its role in informing future research, quantitative studies will be individually assessed for bias and qualitative studies will be individually assessed for methodological rigour.

- A limitation of this review will be the exclusion of unpublished literature for which authors are unable to provide sufficient additional information, and research not published in English.

survivors and 12 million stroke related deaths worldwide in $2030^{3}$. Secondary prevention of stroke is currently inadequate with incidence rates as high as $40 \%$. Secondary strokes tend to be more severe with a mortality rate nearly double that of first stroke. ${ }^{5}$ Effective secondary prevention strategies must be significantly improved to prevent the impact of stroke recurrence.

Lifestyle interventions which involve increasing physical activity in stroke survivors can improve secondary stroke risk factors. ${ }^{6} 7$ Despite this, stroke survivors frequently have low activity levels. ${ }^{8-10}$ Simply providing prompts and encouragement is not sufficient to achieve increases in physical activity in stroke survivors. ${ }^{9}$ The potential importance that supervision of exercise plays in increasing physical activity in stroke survivors is emerging 
in the evidence. Research has found supervised exercise is superior to unsupervised exercise in increasing long-term physical activity levels in stroke survivors. ${ }^{11}$ Furthermore, stroke survivors have identified support provided by qualified staff during supervised exercise was a key facilitator for increasing physical activity. ${ }^{12}$ Supervised exercise can also improve walking ability ${ }^{13}$ mobility, ${ }^{14}$ balance $^{14}$ and endurance $^{14}$ in stroke survivors; all key elements of physical function which are positively associated with physical activity levels in this population. ${ }^{15}$

Barriers to physical activity identified by stroke survivors include transport, ${ }^{1617}$ economic constraints, ${ }^{16}$ distance $^{17}$ and a perceived lack of appropriate services. ${ }^{16}$ Marzolini et al found barriers to physical activity identified in their study also increased with increased socioeconomic disadvantage. ${ }^{17}$ Telehealth uses 'telecommunications and virtual technology to deliver health care outside of traditional health care facilities'. ${ }^{18}$ This rapidly evolving mode of service delivery has significant potential to improve equity of service delivery and overcome barriers such as access, distance, cost and transport. Evidence is emerging across chronic conditions regarding the application of supervised exercise delivered via telehealth. ${ }^{19-24}$ Preliminary research indicates these interventions may be feasible,${ }^{21}$ improve self-efficacy ${ }^{20}$ and reduce costs of exercise programme delivery. ${ }^{24}$

Stroke-related impairment may impact the safety and efficacy of supervised exercise delivered via telehealth to reduce secondary stroke risk factors. Stroke survivors are commonly affected by impaired cognition, ${ }^{25}$ physical ability ${ }^{2627}$ and poststroke fatigue. ${ }^{28}$ Internationally, the potential for supervised exercise to optimise stroke survivor safety is recognised. The American physical activity and exercise recommendations for stroke survivors ${ }^{29}$ and the Canadian stroke best practice recommendations: Secondary prevention of stroke ${ }^{30}$ suggest the consideration of health professional supervised exercise where co-morbidity, ${ }^{29} 30$ falls risk ${ }^{30}$ and level of disability ${ }^{29}$ present a risk. Seventy-nine percent of stroke survivors are reported to have at least one cardiovascular co-morbidity, while over a quarter have at least two. ${ }^{31}$ Falls are common post stroke ${ }^{32-34}$ and $45 \%$ of community dwelling stroke survivors have been found to fall at least once in a 12-month period. ${ }^{34}$ Supervised exercise delivered via telehealth may provide a safe and accessible way to increase physical activity for stroke survivors at risk of falls or cardiac events. Preliminary evidence has found telehealth rehabilitation for improving motor function in stroke is at least as effective as its centre-based equivalent. ${ }^{35}$ However, the safety and efficacy of supervised exercise training for the reduction of secondary stroke risk factors (including aerobic and resistance training) delivered via telehealth remains largely unexplored..$^{35}$

Focusing on supervised exercise due to its significant potential to support increased physical activity in stroke survivors, our scoping review will map the available evidence surrounding supervised exercise delivered via telehealth in chronic conditions. This will provide an understanding of current evidence to inform clinical trials in stroke. Our specific research questions are as follows.

With regard to interventions involving supervised exercise delivered via telehealth in chronic conditions:

1. What population groups have been included in the research and what are the key characteristics of the interventions delivered (including: frequency, duration and intensity; types of exercise included and telehealth modalities used)?

2. What are health professionals', participants' and carers' experiences of, or attitudes towards, supervised exercise delivered via telehealth?

3. What strategies have been used to optimise safety, feasibility, delivery and adherence?

4. What are the barriers and limitations to these interventions and what strategies have been used to mitigate these?

5. What is the effectiveness and cost-effectiveness of telehealth-supervised exercise sessions for reducing secondary stroke risk factors?

\section{METHODS AND ANALYSIS}

Scoping reviews enable the mapping of research findings and identification of gaps in research evidence while providing a source of knowledge translation. ${ }^{36-38} \mathrm{We}$ have chosen the scoping review method to evaluate the evidence surrounding supervised exercise delivered via telehealth in chronic conditions because scoping reviews are suited to areas of research where outcomes are not well established ${ }^{37}$ or are heterogeneous in nature. ${ }^{38} \mathrm{We}$ will use the framework of scoping reviews first described by Arksey and $\mathrm{O}^{\prime}$ Malley ${ }^{36}$ which requires identifying the research question; identifying relevant studies; study selection; charting of the data; and collating, summarising and reporting the results. The refinements to the Arksey and O'Malley ${ }^{36}$ framework suggested by Levac et $a l^{37}$ and Peters $e t a l^{39}$ will also be considered to optimise transparency and methodological rigour.

To optimise reporting this scoping review protocol uses the Preferred Reporting Items for Systematic Reviews and Meta-analyses Protocols (PRISMA-P) ${ }^{40} 41$ method (online supplementary appendix 1). Since not all items in the PRISMA-P are relevant to scoping reviews, we have adapted the items with reference to the PRISMA extension for Scoping Reviews (PRISMA-ScR) ${ }^{38}$ where needed. Our scoping review was initiated on 1 September 2018 and is to be completed by 30 May 2019.

\section{Identifying the research question}

The five research questions of our review are identified in the introduction of this paper. These questions will inform future research into supervised exercise in stroke survivors by mapping and identifying gaps in the available evidence regarding supervised exercise delivered via telehealth in chronic conditions. 
Table 1 Study selection criteria

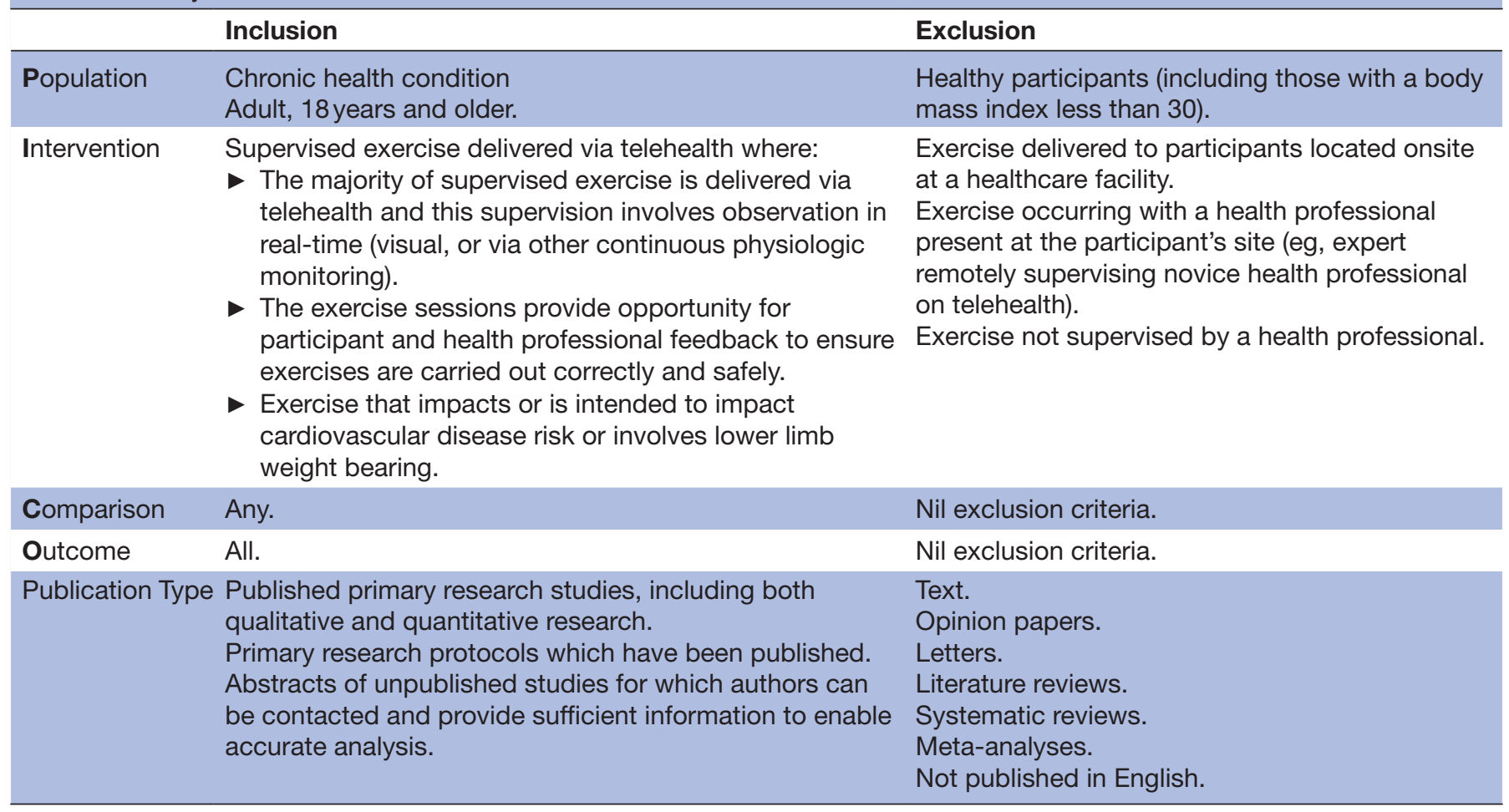

We define the key terms of the scoping review's questions as follows:

- Supervision: real-time monitoring (visual, or through other continuous physiologic monitoring such as echocardiogram or heart rate) by a health professional with the opportunity for participants to receive and provide health professionals feedback in real time to ensure the exercise is being carried out safely and correctly.

- Exercise: our review will consider exercise as physical activity targeted at outcomes that impact cardiovascular disease (and secondary stroke) risk factors, including hypertension, hyperlipidemia, dysglycemia or daily physical activity. To ensure our review captures all evidence relating to the research question regarding the safety of supervised exercise delivered via telehealth and its implications for falls risk, the definition of exercise will also include any intervention involving lower limb weight bearing, for example balance training.

- Telehealth: The application of telecommunications and virtual technology to provide healthcare outside of conventional healthcare facilities (WHO, 2018). ${ }^{18}$

\section{Identifying relevant studies}

Studies will be included in the review if they meet the eligibility requirements set out in table 1 .

All published primary research studies will be included in the review. The exclusion criteria includes otherwise healthy participants with a body mass index (BMI) $<30$ to ensure a consistent definition of the chronic condition obesity as a BMI of 30 or greater. ${ }^{42}$ In order to accurately capture current approaches to real-time supervised exercise delivered via telehealth in this rapidly evolving field, we will also include published trial protocols and abstracts of unpublished studies for which authors can be contacted to provide sufficient information. Studies included in the scoping review will not be limited by year of publication.

\section{Study selection}

We will conduct a comprehensive, systematic search of Medline, CINAHL, Scopus, Cochrane, Pedro and Embase databases. The search strategy will be developed in consultation with a senior research librarian and will include use of the relevant index terms and keywords for 'exercise' and 'telehealth'. We will hand search the reference lists of all included studies as well as relevant systematic reviews. Experts in the field will be contacted to identify any other pertinent research. We will contact authors of abstracts of unpublished studies retrieved and request they provide information to enable accurate analysis of their research.

Full details of the databases' draft search strategies are shown in online supplementary appendix 2.

Studies identified through the search strategy will be exported from each database to EndNote X8.2 and then exported to covidence for removal of duplicates and screening of titles and abstracts. Covidence will be used to manage data throughout the remainder of the review. All titles and abstracts will be independently reviewed by two authors and conflicts will be resolved by a third author. To optimise the reliability of screening of titles and abstracts all authors will participate in the screening of the initial 150 studies for inclusion using the selection criteria 
(outlined in table 1). The authors will then then meet to resolve any issues or ambiguities found in the criteria. Following title and abstract screening, full texts of potentially relevant studies will be assessed for eligibility by two authors independently, with any discrepancies resolved by a third author. To support the inherently iterative nature of scoping reviews, ${ }^{36-39}$ authors will communicate regularly to discuss the selection process. Any refinements made to the selection criteria will be recorded. The selection process will be reported using a PRISMA 2009 Flow diagram. ${ }^{43}$

\section{Data charting}

Data charting is the method used for extracting data in scoping reviews. ${ }^{36} 38$ It allows researchers to capture a breadth of information including detail on processes to provide further context to the research outcomes. ${ }^{36} \mathrm{We}$ will develop a standardised electronic form to chart the data. All authors will pilot the data charting form with the initial five studies to ensure the data extracted optimally addresses the research questions ${ }^{37}$ and then meet to discuss and resolve any issues identified. This process will also facilitate the reliability of the data charting. The remainder of the data will be charted by one author and checked by a second author. Any discrepancies will be resolved by a third author. The iterative nature of scoping reviews means the data charting form may require adaptation during the data charting process. ${ }^{36-39}$ Regular communication between authors will occur to identify, in a timely manner, any need for modification to the data charting form. All significant alterations to the data chart will be recorded.

To avoid inclusion of duplicate data we will identify and group multiple publications relating to the one research project prior to the charting of the data. The study details and outcomes chosen for charting are guided by The Cochrane Collaboration's Checklist of items to consider in data collection or data extraction ${ }^{44}$ and the recommendation of Arksey and O'Malley (2005). ${ }^{36}$ Where available these will include, but are not limited to: bibliographic information; study aims/purpose; research design; number of participants; duration of intervention and follow-up; date; setting; country; co-morbidity; socio-demographics; and specific category of chronic condition. Where available the intervention data extracted will include frequency, intensity, time and type of exercise intervention and any control or comparison groups; the number of intervention groups; the type of telehealth modality used; adherence; satisfaction; and other methods of exercise delivery or support used in the study. All reported outcome measurements will be charted. Details of outcomes which directly inform the research questions including economic viability, intervention feasibility, intervention safety (including adverse events), cardiovascular risk factor indicators (including blood pressure, level of physical activity, cholesterol, lipid profiles, insulin resistance) will be prioritised. Any other key findings or recommendations not captured through the above process which specifically relate to our research questions will also be charted.

All papers included in the scoping review will be critically appraised. We have chosen to undertake critical appraisal for two reasons. The first, to facilitate accurate identification of evidence gaps which Brien et al highlights can be difficult without the assessment of evidence quality. ${ }^{45}$ The second, is to optimise recommendations made for practice to ensure they are based on sound evidence. ${ }^{39}$ We will use The Cochrane Collaboration's tool for assessing risk of bias ${ }^{44}$ to assess the bias of each individual, quantitative study. We will use the Consolidated Criteria for Reporting Qualitative Research checklist ${ }^{46}$ and the Mixed Methods Appraisal Tool (MMAT) - Version $2011^{4748}$ to individually assess qualitative and mixed methods studies for methodological rigour respectively. This critical appraisal will be carried out by two independent authors who will meet to discuss and resolve any discrepancies found in their assessments, with adjudication by a third author if necessary.

\section{Collating summarising and reporting of results}

Our scoping review will be reported using the PRISMA-ScR. ${ }^{38}$ The results will be summarised and reported to prioritise the findings relevant to the specific research questions. Quantitative data and the results of individual studies' critical appraisal will be presented in tabular format. Qualitative data will be analysed thematically and collated concisely into a tabular format. If needed, further narrative description will be provided to aid interpretation of the results. Visual or diagrammatic representation of data will occur to aid its summary or conceptualization as needed.

To aid the synthesis of the results we will provide a narrative summary of the findings most pertinent to the review's research questions. Knowledge gaps in the research evidence and their implications will also be recognised through a narrative summary. Our key findings, informed by the critical appraisal of individual studies, will be used to make recommendations for future research and practice relating to supervised exercise delivered via telehealth.

The breadth of research evidence included in this review enables the comprehensive mapping of interventions involving supervised exercise delivered via telehealth aimed at reducing cardiovascular disease risk factors. As such, caution should be taken when interpreting the findings for individual patient populations. Another potential limitation of the study is the oversight of relevant papers due to the exclusion of grey literature. This has been done to ensure research quality can be assessed to optimise recommendations for practice. These and any further limitations identified during the scoping review process will be acknowledged.

\section{Patient and public involvement}

There will be no patient or public involvement in our scoping review. 


\section{Ethics and dissemination}

The findings of our scoping review will be disseminated through presentation at appropriate forums or conferences. The completed scoping review will also be submitted for publication in a peer reviewed journal and form part of a $\mathrm{PhD}$ thesis. Findings will be directly translated to inform the development of a supervised exercise programme delivered via telehealth that will be pilot tested and evaluated in terms of effect on reducing secondary stroke risk factors. We will use only secondary de-identified data in the scoping review, therefore ethics approval is not required.

\section{DISCUSSION}

The high mortality rates and the significant burden of disease resulting from secondary stroke must be addressed. Our review will explore emerging research in relation to the efficacy, acceptability, safety, economics and feasibility of supervised exercise delivered via telehealth. This research has the potential to provide strategies to overcome current barriers in the translation of evidence for physical activity in stroke survivors to reduce stroke recurrence.

This review will significantly contribute to the knowledge base of exercise and rehabilitation delivered via telehealth. The breadth of research captured means it has implications beyond stroke care to broadly inform the application of supervised exercise and rehabilitation via telehealth. It is anticipated that our findings will be relevant to researchers, healthcare workers and policy-makers at a national and international level .

\section{Author affiliations}

${ }^{1}$ School of Health Sciences, University of Newcastle, Newcastle, New South Wales, Australia

${ }^{2}$ Physiotherapy, Western Health, St Albans, Victoria, Australia

${ }^{3}$ Physiotherapy, University of Melbourne, School of Health Sciences, Melbourne,

Victoria, Australia

${ }^{4}$ Physiotherapy, La Trobe University, Bundoora, Victoria, Australia

${ }^{5}$ Adelaide Nursing School, University of Adelaide, Adelaide, South Australia, Australia ${ }^{6}$ Physiotherapy, The University of Melbourne, Parkville, Victoria, Australia ${ }^{7}$ Florey Institute of Neuroscience and Hunter Medical Research Institute, Centre for Research Excellence in Stroke Recovery and Rehabilitation, Newcastle, Australia

Acknowledgements The authors would like to acknowledge the contribution of Ms Debbie Booth for her assistance and advice in drafting the search strategy.

Contributors All authors made significant intellectual contributions to the protocol. CE conceived the idea for the scoping review. ERR (guarantor) and CE conceptualised and drafted the research questions and study selection criteria, while CMS, EAL, NAF and AP contributed to their further development. ERR developed the study methodology with EAL, CMS, NAF, AP and CE providing feedback for refinement. All authors contributed to the drafting and editing, and approved the final manuscript.

Funding Emily Ruth Ramage was supported by an Australian Government Research Training Program Scholarship. Associate Professor Coralie English was supported by a National Heart Foundation Future Leaders Fellowship (\#101177). Dr Elizabeth A Lynch was supported by a National Health and Medical Research Council Early Career Research Fellowship (\#1138515).

Competing interests ERR reports support from an Australian Government Research Training Program Scholarship. CE reports support from the National
Heart Foundation of Australia and EAL reports support from the National Health and Medical Research Council.

Patient consent for publication Not required.

Provenance and peer review Not commissioned; externally peer reviewed.

Open access This is an open access article distributed in accordance with the Creative Commons Attribution Non Commercial (CC BY-NC 4.0) license, which permits others to distribute, remix, adapt, build upon this work non-commercially, and license their derivative works on different terms, provided the original work is properly cited, appropriate credit is given, any changes made indicated, and the use is non-commercial. See: http://creativecommons.org/licenses/by-nc/4.0/.

\section{REFERENCES}

1. Organization WH. Health statistics and information systems: Disease burden and mortality estimates, cause-specific mortality 2000-2016 [Internet]. World Health Organization. 2018 http://www.who.int/ healthinfo/global_burden_disease/estimates/en/ (Accessed $19 \mathrm{Sep}$ 2018).

2. World Health Organisation. Health statistics and information systems: Disease burden and mortality estimates, Disease burden 20002016 [Internet]: World Health Organisation, 2018. http://www.who. int/healthinfo/global_burden_disease/estimates/en/index1.html. (Accessed 19 Sep 2018).

3. Feigin VL, Forouzanfar MH, Krishnamurthi R, et al. Global and regional burden of stroke during 1990-2010: findings from the Global Burden of Disease Study 2010. Lancet 2014;383:245-55.

4. Mohan KM, Wolfe CD, Rudd AG, et al. Risk and cumulative risk of stroke recurrence: a systematic review and meta-analysis. Stroke 2011;42:1489-94.

5. Jørgensen HS, Nakayama H, Reith J, et al. Stroke recurrence: predictors, severity, and prognosis. The copenhagen stroke study. Neurology 1997;48:891-5.

6. Deijle IA, Van Schaik SM, Van Wegen EE, et al. Lifestyle interventions to prevent cardiovascular events after stroke and transient ischemic attack: Systematic review and meta-analysis. Stroke 2017;48:174-9.

7. D'Isabella NT, Shkredova DA, Richardson JA, et al. Effects of exercise on cardiovascular risk factors following stroke or transient ischemic attack: a systematic review and meta-analysis. Clin Rehabil 2017;31:1561-72.

8. English C, Healy GN, Coates A, et al. Sltting and activity time in people with stroke. Phys Ther 2016;96:193-201.

9. Boysen G, Krarup LH, Zeng X, et al. ExStroke Pilot Trial of the effect of repeated instructions to improve physical activity after ischaemic stroke: a multinational randomised controlled clinical trial. $\mathrm{BMJ}$ 2009;339:b2810.

10. Fini NA, Holland AE, Keating J, et al. How physically active are people following stroke? Systematic review and quantitative synthesis. Phys Ther 2017;97:707-17.

11. Olney SJ, Nymark J, Brouwer B, et al. A randomized controlled trial of supervised versus unsupervised exercise programs for ambulatory stroke survivors. Stroke 2006;37:476-81.

12. Simpson LA, Eng JJ, Tawashy AE. Exercise perceptions among people with stroke: Barriers and facilitators to participation. Int $J$ Ther Rehabil 2011;18:520-9.

13. English C, Hillier SL, Lynch EA. Circuit class therapy for improving mobility after stroke. Cochrane Database Syst Rev 2017;6:CD007513.

14. Duncan P, Studenski S, Richards L, et al. Randomized clinical trial of therapeutic exercise in subacute stroke. Stroke 2003;34:2173-80.

15. Thilarajah S, Mentiplay BF, Bower KJ, et al. Factors associated with post-stroke physical activity: A systematic review and meta-analysis. Arch Phys Med Rehabil 2018;99:1876-89.

16. Nicholson SL, Donaghy M, Johnston M, et al. A qualitative theory guided analysis of stroke survivors' perceived barriers and facilitators to physical activity. Disabil Rehabil 2014;36:1857-68.

17. Marzolini S, Balitsky A, Jagroop D, et al. Factors Affecting Attendance at an Adapted Cardiac Rehabilitation Exercise Program for Individuals with Mobility Deficits Poststroke. J Stroke Cerebrovasc Dis 2016;25:87-94.

18. World Health Organisation. Health and sustainable development: Telehealth [Internet]. ND: World Health Organization. http://www.who. int/sustainable-development/health-sector/strategies/telehealth/en/. (Accessed 19 Sep 2018).

19. van Egmond MA, van der Schaaf M, Vredeveld $T$, et al. Effectiveness of physiotherapy with telerehabilitation in surgical patients: a systematic review and meta-analysis. Physiotherapy 2018;104:277-98. 
20. Tsai LL, McNamara RJ, Moddel C, et al. Home-based telerehabilitation via real-time videoconferencing improves endurance exercise capacity in patients with COPD: The randomized controlled TeleR Study. Respirology 2017;22:699-707.

21. Paneroni M, Colombo F, Papalia A, et al. Is telerehabilitation a safe and viable option for patients with copd? A feasibility study. COPD 2015;12:217-25.

22. Hwang R, Bruning J, Morris $\mathrm{N}$, et al. A systematic review of the effects of telerehabilitation in patients with cardiopulmonary diseases. J Cardiopulm Rehabil Prev 2015;35:380-9.

23. Clark RA, Conway A, Poulsen V, et al. Alternative models of cardiac rehabilitation: a systematic review. Eur J Prev Cardiol 2015;22:35-74.

24. Maddison R, Rawstorn JC, Stewart RAH, et al. Effects and costs of real-time cardiac telerehabilitation: randomised controlled noninferiority trial. Heart 2019;105.

25. Douiri A, Rudd AG, Wolfe CD. Prevalence of poststroke cognitive impairment: South London Stroke Register 1995-2010. Stroke 2013;44:138-45.

26. Blennerhassett JM, Levy CE, Mackintosh A, et al. One-Quarter of People Leave Inpatient Stroke Rehabilitation with Physical Capacity for Community Ambulation. J Stroke Cerebrovasc Dis 2018;27:3404-10.

27. Deloitte Access Economics. The economic impact of stroke in Australia. 2013.

28. Nadarajah M, Goh H-T. Post-stroke fatigue: a review on prevalence, correlates, measurement, and management. Top Stroke Rehabil 2015;22:208-20.

29. Billinger SA, Arena R, Bernhardt J, et al. Physical activity and exercise recommendations for stroke survivors: a statement for healthcare professionals from the American Heart Association/ American Stroke Association. Stroke 2014;45:2532-53.

30. Wein T, Lindsay MP, Côté R, et al. Canadian stroke best practice recommendations: Secondary prevention of stroke, sixth edition practice guidelines, update 2017. Int J Stroke 2018;13:420-43.

31. Jørgensen L, Engstad T, Jacobsen BK. Higher incidence of falls in long-term stroke survivors than in population controls: depressive symptoms predict falls after stroke. Stroke 2002;33:542-7.

32. Tilson JK, Wu SS, Cen SY, et al. Characterizing and identifying risk for falls in the LEAPS study: a randomized clinical trial of interventions to improve walking poststroke. Stroke 2012;43:446-52.

33. Weerdesteyn V, de Niet M, van Duijnhoven $\mathrm{HJ}$, et al. Falls in individuals with stroke. J Rehabil Res Dev 2008;45:1195-213.
34. Wada N, Sohmiya M, Shimizu T, et al. Clinical analysis of risk factors for falls in home-living stroke patients using functional evaluation tools. Arch Phys Med Rehabil 2007;88:1601-5.

35. Sarfo FS, Ulasavets U, Opare-Sem OK, et al. Tele-rehabilitation after stroke: An updated systematic review of the literature. J Stroke Cerebrovasc Dis 2018;27:2306-18

36. Arksey H, O'Malley L. Scoping studies: towards a methodological framework. Int J Soc Res Methodol 2005;8:19-32.

37. Levac D, Colquhoun H, O'Brien KK. Scoping studies: advancing the methodology. Implement Sci 2010;5:69.

38. Tricco AC, Lillie E, Zarin W, et al. PRISMA Extension for Scoping Reviews (PRISMA-ScR): Checklist and Explanation. Ann Intern Med 2018;169:467.

39. Peters MD, Godfrey CM, Khalil H, et al. Guidance for conducting systematic scoping reviews. Int J Evid Based Healthc 2015;13:141-6.

40. Moher D, Shamseer L, Clarke M, et al. Preferred reporting items for systematic review and meta-analysis protocols (PRISMA-P) 2015 statement. Syst Rev 2015;4:1.

41. Shamseer L, Moher D, Clarke M, et al. Preferred reporting items for systematic review and meta-analysis protocols (PRISMA-P) 2015: elaboration and explanation. BMJ 2015;349:g7647.

42. World Health Organization. Obesity and overweight [Fact Sheet of the World Health Organization]. 2018 http://www.who.int/en/newsroom/fact-sheets/detail/obesity-and-overweight (accessed 1 Dec 2018).

43. Moher D, Liberati A, Tetzlaff J, et al. Preferred reporting items for systematic reviews and meta-analyses: the PRISMA statement. BMJ 2009;339:b2535.

44. GS. Higgins J, ed. Cochrane Handbook for Systematic Reviews of Interventions Version 5.1.0 The Cochrane Collaboration, 2011.

45. Brien SE, Lorenzetti DL, Lewis S, et al. Overview of a formal scoping review on health system report cards. Implement Sci 2010;5:2.

46. Tong A, Sainsbury P, Craig J. Consolidated criteria for reporting qualitative research (COREQ): a 32-item checklist for interviews and focus groups. Int J Qual Health Care 2007;19:349-57.

47. Pluye P, Robert E, Cargo M, et al. Proposal: A mixed methods appraisal tool for systematic mixed studies reviews. http://mixedmet hodsappraisaltoolpublic.pbworks.com/. Archived by WebCite cited 2018 Oct 22 http://www.webcitation.org/5tTRTc9yJ2011 (Accessed 22 Oct 2018).

48. Pluye P, Gagnon MP, Griffiths F, et al. A scoring system for appraising mixed methods research, and concomitantly appraising qualitative, quantitative and mixed methods primary studies in Mixed Studies Reviews. Int J Nurs Stud 2009;46:529-46. 